\title{
Evaluación y análisis de la paja canalera como material biomásico para la generación de energía eléctrica mediante gasificación
}

\author{
Assessment of wild sugarcane as biomass material for power \\ generation by gasification
}

\author{
Daniel Navarro ${ }^{l}$, Jaime Lay ${ }^{l}$, Orlando Aguilar ${ }^{2 *}$ \\ ${ }^{1}$ Licenciatura en Ingeniería Mecánica Industrial, Facultad de Ingeniería Industrial, Universidad Tecnológica de Panamá, \\ ${ }^{2}$ Facultad de Ingeniería Mecánica, Universidad Tecnológica de Panamá
}

\begin{abstract}
Resumen La Paja Canalera (Saccharum spontaneum) es una especie herbácea no nativa de la flora panameña. Esta crece en condiciones muy extremas de suelo y se propaga rápidamente, por lo tanto, no permite el desarrollo de otras plantas y en la estación seca, es el objeto principal de incendios forestales espontáneos o intencionales, lo que afecta al medio ambiente y a seres humanos. Este estudio se centra en evaluar el aprovechamiento de la especie como material biomásico para la producción de energía mediante el proceso termoquímico de gasificación, tecnología totalmente limpia e innovadora para el país. Para ello se requiere determinar las características energéticas de la especie y del syngas obtenido, lo cual se podrá realizar a través de la metodología descrita. En la actualidad la Cuenca del Canal de Panamá existen aproximadamente unas 6,931.9 hectáreas cubiertas por dicha especie, las cuales representan unos 6088.18 TJ de energía; estimación basada en estudios previos.
\end{abstract}

Palabras clave Biocombustible, biomasa, conversión termoquímica, energías renovables, gasificación, paja canalera, Saccharum spontaneum, syngas.

\begin{abstract}
Wild Sugarcane (Saccharum spontaneum) is an herbaceous species not native to the Panamanian flora, grows in very extreme conditions of soil and spreads rapidly. Therefore it does not allow the development of other plants. During dry season, wild sugarcane is the main cause of intentional or spontaneous wildfire which affects humans and the environment. This research focuses on evaluating the use of S. spontaneum as a biomass feedstock for power generation through the thermochemical process of gasification, a clean and innovative technology for the country. In order to accomplish this, it is necessary to determine the energetic properties of the species and the syngas obtained, which can be done through the described methodology. Currently, approximately 6,931.9 ha of the Panama Canal Watershed are covered by this species, which represent about 6088.18 TJ of energy; this estimate is based on previous research.
\end{abstract}

Keywords Biofuels, biomass, thermochemical conversion, renewable energy, gasification, wild sugarcane, Saccharum spontaneum, syngas.

* Corresponding Author: orlando.aguilar@utp.ac.pa

\section{Introducción}

Desde la antigüedad el hombre ha utilizado las distintas formas de energía encontradas en la naturaleza para realizar sus actividades diarias y satisfacer sus necesidades, lo cual ha permitido el desarrollo de las civilizaciones a través de los tiempos. Sin embargo, en los últimos años la sociedad se ha hecho altamente dependiente a los combustibles fósiles, haciendo que la migración a otras fuentes de energía no sea una tarea fácil, lo cual aunado a su escasez y a la gran contaminación que producen, se requiere de grandes esfuerzos y nuevos estudios de la comunidad científica para la implementación de formas alternativas de energía que sean renovables y amigables con el medioambiente.
Se entiende como biomasa todo material orgánico derivado de seres vivo (planta o animales) o recientemente muertos, y puede ser utilizado como fuente de energía para ser transformado o utilizado directamente como combustible [1]. La biomasa es un recurso abundante y altamente disponible, la cual, aprovechándola de una forma sostenible, es una de estas fuentes alternativas de energía que puede contribuir a solución de la problemática energética mundial.

Se han desarrollado diversos métodos para la conversión de la energía contenida en la biomasa, uno de ellos es la gasificación, el cual es un proceso termoquímico, en el que el contenido energético de la biomasa, en estado sólido, mediante una serie de reacciones químicas, es transformado a un gas combustible denominado "syngas", el cual puede ser utilizado 
en diversas aplicaciones, de forma similar que algunos combustibles fósiles actualmente utilizados.

En Panamá existe una gran cantidad y diversidad de especies vegetales, lo cual indica el enorme potencial de energía en forma de biomasa que poseemos. La Saccharum spontaneum, comúnmente conocida como Paja Canalera, Paja Blanca o Caña Silvestre, un tipo de planta herbácea originaria de Asia, introducida a nuestro país en la primera mitad del siglo $\mathrm{XX}$; actualmente cubre extensas áreas de terreno en todo el país como lo muestra la "figura 1" [2].

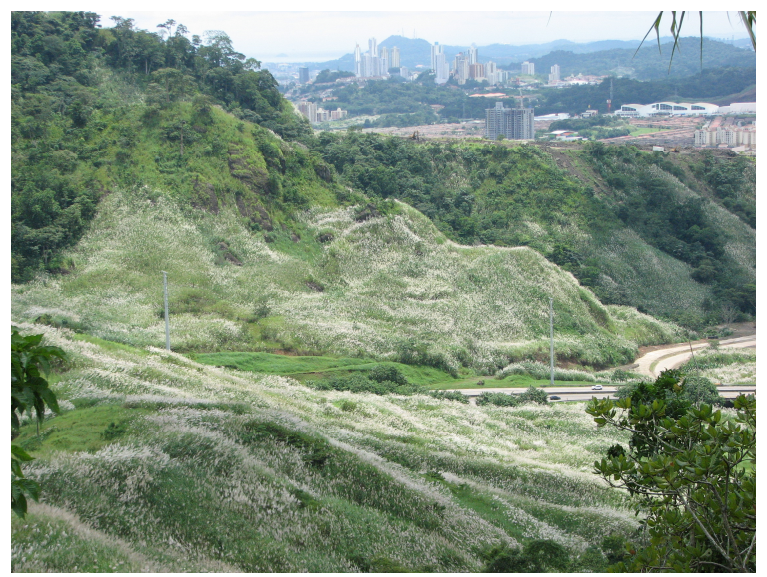

Figura 1. Ejemplo de una extensa área cerca de la ciudad de Panamá cubierta por S. spontaneum.

Esta investigación busca evaluar el desempeño de un sistema de gasificación, utilizando la $S$. spontaneum como material biomásico. Para ello se considera en primera instancia el estudio de sus características y propiedades termofísicas. Por último, la evaluación de la composición y poder calorífico del syngas obtenido, estimando la viabilidad de la utilización de esta tecnología como alternativa energética en nuestro país para la generación de electricidad.

\section{Planteamiento del problema}

En Panamá, comparado con otros países, lastimosamente no estamos muy avanzados en el tema del aprovechamiento de los recursos energéticos alternativos, como los recursos biomásicos que a simple vista son abundantes y altamente disponibles, donde solo el $0.24 \%$ ( $8.1 \mathrm{MW}$ ) de la generación eléctrica en el país proviene de recursos biomásicos, según informes de la Secretaria Nacional de Energía [3].

Actualmente la $S$. spontaneum cubre extensas áreas de terreno en todo el país y es considerada una plaga, debido a su rápida germinación, diseminación y crecimiento, lo cual imposibilita el desarrollo natural de la flora autóctona. Esta especia sobrevive en ambientes críticos y germina en suelos pobres. Durante la época seca es el principal objeto de quema provocada o espontánea, afectando a seres humanos y al medio ambiente, sin embargo, se muestra resistente y rebrota rápidamente [4].

Esta especie, al representar un serio problema medioambiental y contar con la disponibilidad de grandes extensiones de terreno densamente pobladas por la misma, surge el planteamiento de realizar esta investigación con el objeto de evaluar un sistema de gasificación utilizando la Paja Canalera como materia prima, determinando las características energéticas de la especie y del syngas obtenido luego de dicho proceso. Esto nos permitirá analizar el potencial del aprovechamiento energético de la $S$. spontaneum en nuestro país.

Bajo este marco surgen los siguientes cuestionamientos ¿La República de Panamá está contemplando el aprovechamiento de recursos biomásicos como fuente de energía? ¿Es aprovechada actualmente la Paja Canalera de alguna forma? ¿Puede la $S$. spontaneum ser una fuente de energía factible y rentable en Panamá? ¿Existen tecnologías que pudieran ser factibles para el aprovechamiento energético de la Paja Canalera? ¿Qué tipo de sistema de gasificación será el adecuado para explotar el poder calorífico de la Paja Canalera? ¿A qué escala? ¿Ayudará este aprovechamiento a la erradicación de esta especie o a la disminución de sus efectos negativos sobre el medioambiente? Estos cuestionamientos incentivan a la duda y el deseo de investigación para encontrarle provecho a dicha especie herbácea considerada como maleza y plaga en nuestro país.

\section{Metodología}

\subsection{Muestreo}

El estudio se enmarcará en la Cuenca Hidrográfica del Canal de Panamá (CHCP), ya que contamos con mayor información para esta región, gracias al apoyo proporcionado por la División de Ambiente de la Autoridad del Canal de Panamá. Con la ayuda del mapa de Cobertura vegetal y Uso de Suelo 2013 pertenecientes al último "Informe de Cobertura Vegetal y Uso de Suelo de la CHCP" [5] se identificaron las extensiones de territorio dominados por $S$. spontaneum y se seleccionaron sitios posibles de muestreo, evaluando la accesibilidad de los mismos, para luego escoger aleatoriamente cuatro sitios finales de muestreo. Se determinó aleatoriamente dentro de los sitios seleccionados una parcela con un área de 4 $\mathrm{m}^{2}(2 \mathrm{~m} \times 2 \mathrm{~m})$. Una vez en el sitio se determinaron las coordenadas y se registró una descripción de las características del suelo y la configuración del terreno. Se midió el tamaño de las plantas desde el suelo hasta la copa de las hojas en cada esquina y en el centro de la parcela con una cinta métrica. Se escogió un segmento de $1 \mathrm{~m}^{2}$ dentro de la parcela, donde se contaron los tallos, para luego escoger diez de ellos al azar, para medir el diámetro basal y la altura de los mismos. El diámetro basal se midió, con un calibrador de vernier, justo encima del primer nódulo; y la altura del tallo, desde la base 
hasta el último nódulo como se muestra en la "figura 2". Se cortan todos los tallos dentro del área de muestra y se colocan en una lona para ser transportados al sitio de almacenamiento. Se pican a un tamaño de partícula de $1 \mathrm{~cm}$ y se pesa todo el material recolectado $\left(\mathrm{m}_{\mathrm{rec}}\right)$. Se seca al aire hasta que no varíe su peso $\left(m_{\text {sec.aire }}\right)$. Para ello se seleccionará una pequeña muestra de control la cual se pesará periódicamente. Con esto se determina el contenido de humedad ambiental o externa de la biomasa recolectada, como lo muestra en la ecuación (1).

$$
\% H=\frac{m_{r e c}-m_{\text {sec.aire }}}{m_{\text {rec }}} \cdot 100
$$
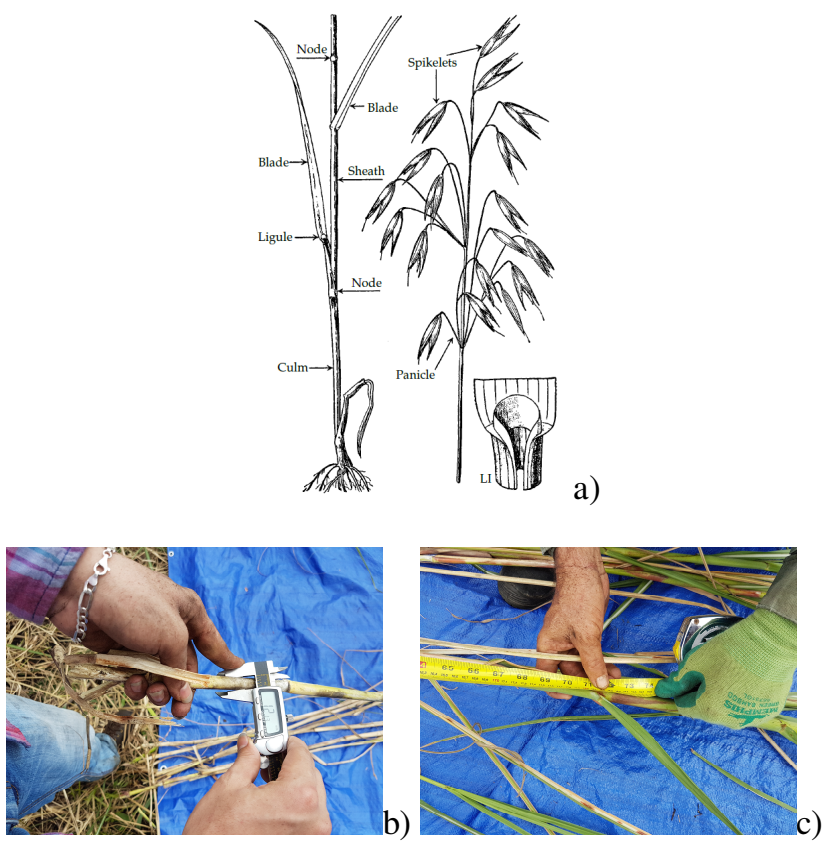

Figura 2. Caracterización de los tallos de las plantas en la recolección, a) estructura típica de una planta herbácea [6], b) medición del diámetro basal, c) medición de la altura del tallo.

\subsection{Propiedades Termofísicas}

Para hacer una descripción termofísica de la biomasa, se escogieron pequeñas muestras de $50 \mathrm{~g}$, representativas de todo el material recolectado y se desarrolló un análisis próximo, en el cual se determina el contenido de humedad, cenizas, materia volátil y carbón fijo expresados en porcentaje en peso del material, para ello se utilizan los procedimientos descritos en las normas ASTM E-1757, ASTM E1756 y ASTM E-1755 [79]. Esta descripción de la biomasa es muy importante para poder determinar y predecir el comportamiento del sistema de gasificación a utilizar [10]. Luego se determina el poder calorífico mediante una bomba calorimétrica IKA C2000.

Para efectos del manejo y transporte de la biomasa en el sistema de gasificación es importante determinar algunas otras propiedades físicas. La fluidez de la biomasa es importante para determinar el sistema de alimentación en un sistema de gasificación. Para ello se determina el ángulo de reposo que no es más que el ángulo promedio medido desde un plano horizontal que forman las partículas de biomasa apiladas de forma aleatoria [11]. Con este fin se llena un tubo con las partículas de biomasa reposando sobre una superficie plana, el tubo se levanta haciendo que las partículas formen una pila y se mide el respectivo ángulo. La densidad aparente es la medida de la relación de la masa del material al volumen total ocupado, incluyendo la porosidad y espacios entre las partículas del material, para ello se deja caer las partículas de biomasa en una caja de tamaño estándar (305x305x305 mm) desde una altura de $610 \mathrm{~mm}$ siguiendo el procedimiento descrito en la norma ASTM E873 [12, 13].

\subsection{Gasificación}

Una vez que se ha caracterizado el material biomásico, mediante la evaluación de las propiedades termofísicas, se puede escoger el tipo de sistema de gasificación que mejor se desempeñe ante dicho material biomásico a utilizar, refiriéndonos a las recomendaciones dadas en estudios previos [1] [9-11] [14-15].

Por último, mediante un analizador de gases Cubic Ruiyi Gasboard 3100P, se puede registrar la composición química del syngas $\left(\mathrm{CO}, \mathrm{CO}_{2}, \mathrm{CH}_{4}, \mathrm{H}_{2}, \mathrm{O}_{2}\right.$ y $\left.\mathrm{C}_{\mathrm{x}} \mathrm{H}_{\mathrm{y}}\right)$ y su poder calorífico. Con estos últimos datos se evalúa la eficiencia del sistema de gasificación, ecuación (2).

$$
\eta_{\text {gasificador }}=\frac{m_{\text {gas }} \cdot P C I_{\text {gas }}}{m_{\text {biomasa }} \cdot P C I_{\text {biomasa }}}
$$

Donde, $\mathrm{m}_{\text {gas }}$ es masa del gas producido, $\mathrm{PCI}_{\text {gas }}$ el poder calorífico inferior, $\mathrm{m}_{\text {biomasa }}$ es la masa de la biomasa en el gasificador y $\mathrm{PCI}_{\text {biomasa }}$ el poder calorífico inferior de la biomasa.

\section{Resultados}

Al momento de la redacción de este artículo, los resultados preliminares han arrojado los sitios de muestreo. Para ello se preseleccionaron diez sitios, atendiendo a la accesibilidad y configuración del terreno. Por motivos de seguridad, esto se hizo utilizando el Mapa de Cobertura de Paja Blanca en la CHCP al 2013 [5] observando los puntos a simple vista con alta densidad. Al azar se seleccionaron finalmente cuatro sitios, los cuales se muestran en la figura 3 y en la tabla 1 .

Un estudio realizado en 2009 en áreas de la CHCP determinó que en una parcela infestada por $S$. spontaneum presentaba en el mes de abril una densidad superficial de biomasa de 5630.0 $\pm 1996.9 \mathrm{~g} / \mathrm{m}^{2}$ [4]. Este dato es de mucha importancia al dar luces del rendimiento biomásico por unidad de superficie de la especie encontrada en estado natural, lo cual también pretende determinar este trabajo. Sin embargo, el estudio anteriormente mencionado incluyó solamente un sitio de muestreo, ya que los objetivos del mismo eran diferentes; por ello el nuestro, 
considera al menos cuatro sitios considerando las variaciones de las condiciones de crecimiento para la especie de un sitio a otro dentro de la CHCP.

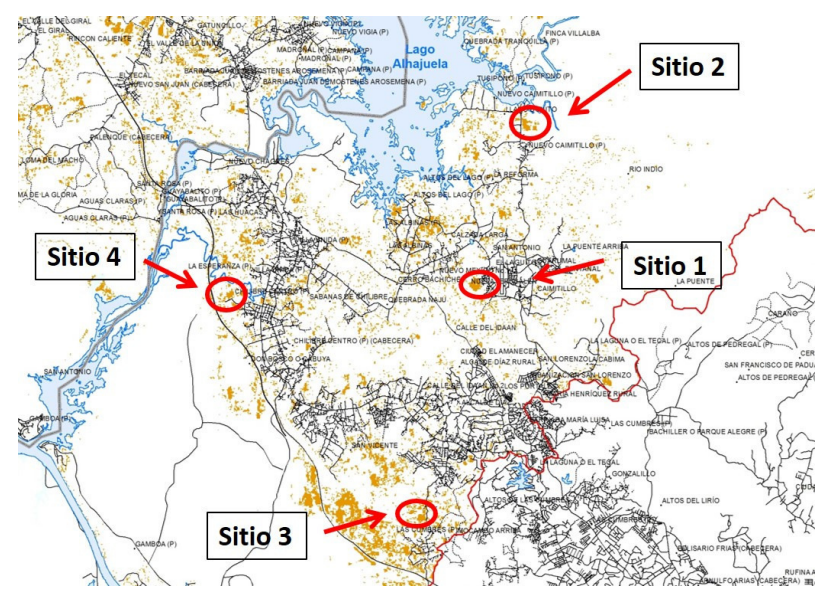

Figura 3. Ubicación de los sitios de muestreo seleccionados dentro de la CHCP. Fuente [5].

Tabla 1. Sitios seleccionados para el muestreo

\begin{tabular}{|c|l|l|}
\hline Sitio & \multicolumn{1}{|c|}{ Descripción } & \multicolumn{1}{c|}{ Coordenadas } \\
\hline 1 & Los Pinos - Calzada Larga, Chilibre & $\begin{array}{l}9^{\circ} 09^{\prime} 59.5^{\prime \prime} \mathrm{N} \\
79^{\circ} 33^{\prime} 16.13^{\prime \prime} \mathrm{O}\end{array}$ \\
\hline 2 & Nuevo Caimitillo, Chilibre & $\begin{array}{l}9^{\circ} 12^{\prime} 50^{\prime \prime} \mathrm{N} \\
79^{\circ} 32^{\prime} 15.1^{\prime \prime} \mathrm{O}\end{array}$ \\
\hline 3 & Villa Grecia Adentro, Las Cumbres & $\begin{array}{l}9^{\circ} 05^{\prime} 15.1^{\prime \prime} \mathrm{N} \\
79^{\circ} 34^{\prime} 11.3^{\prime \prime} \mathrm{O}\end{array}$ \\
\hline 4 & Chilibre Centro, Chilibre & $\begin{array}{l}9^{\circ} 09^{\prime} 34.8^{\prime \prime} \mathrm{N} \\
79^{\circ} 37^{\prime} 56.5^{\prime \prime} \mathrm{O}\end{array}$ \\
\hline
\end{tabular}

El último "Informe de Cobertura Vegetal y Uso de Suelo de la Cuenca Hidrográfica del Canal de Panamá" publicado en el año 2013, refleja que unas 6931.93 ha de la CHCP están infestadas de S. spontaneum, lo cual representa un $2.02 \%$ de la superficie de la misma [5]. Esta información es útil para el cálculo del potencial energético total en la $\mathrm{CHCP}$, conociendo el poder calorífico de la misma $(\mathrm{MJ} / \mathrm{kg})$ y el rendimiento biomásico por superficie ( $\mathrm{kg} / \mathrm{ha})$.

Se espera que la paja canalera presente propiedades similares a otras especies herbáceas perennes de ciclo C4 reflejadas en estudios previos [12] [14] [16]. Consentino et al (2013) [17] determinó, mediante un análisis elemental (CHN), el poder calorífico inferior $(\mathrm{PCI})$ de la S. spontaneum bajo diferentes condiciones de irrigación. Una de las parcelas cultivadas de S. spontaneum que presentó unos $972.5 \mathrm{~mm}$ de agua disponible, lo más cercano a nuestro clima con unos 2200 $\mathrm{mm}$ de lluvia al año en promedio [18], encontrándose los siguientes resultados mostrados en la tabla 2.
Tabla 2. Propiedades termoquímicas de la S. spontaneum

\begin{tabular}{|c|c|c|c|}
\hline \multicolumn{2}{|c|}{ Análisis próximo } & \multicolumn{2}{c|}{ Análisis último } \\
\hline Humedad (\%w/w) & 49.5 & $\mathrm{C}(\% \mathrm{w} / \mathrm{w})$ & 43.76 \\
\hline Cenizas $(\% \mathrm{w} / \mathrm{w})$ & 4.8 & $\mathrm{H}(\% \mathrm{w} / \mathrm{w})$ & 5.80 \\
\hline & & $\mathrm{N}(\% \mathrm{w} / \mathrm{w})$ & 0.40 \\
\hline & & $\mathrm{PCI}(\mathrm{MJ} / \mathrm{kg})$ & 15.60 \\
\hline
\end{tabular}

Con dicho poder calorífico y el rendimiento biomásico de los estudios anteriormente mencionados, podemos realizar la estimación que en la CHCP existen aproximadamente unos 6088.18 TJ de energía aprovechables provenientes de la conversión termoquímica de la paja canalera. Si se cosechara 1 hectárea de paja canalera para alimentar a una planta de generación eléctrica por un año ( 8760 horas) de forma continua se podría alimentar a una carga de $27.85 \mathrm{~kW}$, lo cual es suficiente para abastecer la necesidad de energía eléctrica a una pequeña comunidad.

\section{Conclusiones}

1. La gasificación, así como muchas otras alternativas innovadoras para la producción de energía limpia no han sido ampliamente desarrolladas en Panamá, en especial las tecnologías de biomasa, las cuales representan actualmente solo el $0.24 \%$ de producción de energía eléctrica, a pesar de que contamos con un enorme potencial para las mismas.

2. La S. spontaneum, así como muchos otros materiales biomásicos disponibles en la República de Panamá, carecen de estudios para el análisis y evaluación de su aprovechamiento energético. Con lo cual, estudios como este, pueden realizar un gran aporte al desarrollo energético limpio y sostenible de Panamá.

3. La CHCP contiene unas 6931.9 ha cubiertas por paja canalera, lo cual se traduce a una disponibilidad de energía de 6088.18 TJ, de la cual una importante fracción es desperdiciada en quemas. Esta energía puede ser aprovechada en sistemas de conversión termoquímica como la gasificación, evidenciado en numerosos estudios con especies similares.

4. La cosecha de 1 ha de S. spontaneum puede alimentar por un año a una pequeña planta de generación de 27.85 $\mathrm{kW}$, la cual produciría la energía suficiente para un pequeño poblado; un escenario que la convierte en una opción interesante para aplicaciones a pequeña escala en sitios rurales o remotos con gran disponibilidad del recurso.

\section{AGRADECIMIENTO}

Agradecemos al Dr. Cecilio Hernández y al Ing. Ernesto Escobar por su asistencia técnica y guía para realizar todos los experimentos necesarios y a el Laboratorio de Análisis 
Industriales y Ciencias Ambientales (LABAICA), por permitir el uso de sus equipos e instalaciones para el desarrollo experimental.

Agradecemos al Lic. Leopoldo Manso y al Centro de Producción e Investigaciones Agroindustriales (CEPIA) por permitir el uso de su laboratorio y equipo.

Agradecemos al departamento de Metal Mecánica en especial al Sr. Fernando por el apoyo brindado al desarrollo de este trabajo de investigación.

Agradecemos al Laboratorio de Ensayo de Materiales (LEM).

Agradecemos al Laboratorio de Geotecnia (LABGEO).

Agradecemos a la Secretaria Nacional de Ciencias y Tecnología (SENACYT) por el apoyo brindado y el financiamiento de este trabajo de investigación.

\section{REFERENCIAS}

[1] Chris Higman, Maarten van der Burgt, Gasification, Gulf Professional Publishing, Burlington, USA, 2003.

[2] Arturo Cerezo, "Antecedentes del origen y objetivo de la introducción de la maleza paja blanca (Saccharum spontaneum L.) a Panamá", Autoridad del Canal de Panamá, Sección de Manejo de Cuenca, pp 1-6, marzo de 2010.

[3] Secretaría Nacional de Energía, "El Mercado Eléctrico de la República de Panamá", 31 de diciembre de 2016. Disponible en línea: http://www.energia.gob.pa/Electricidad

[4] Kristin Saltonstall, Graham Bonnett, "Fire promotes growth and reproduction of Saccharum spontaneum (L.) in Panama", Springer Netherlands, Biological Invasion, vol. 19, no. 12, pp. 2479-2488, 2012.

[5] Autoridad del Canal de Panamá, "Informe de Cobertura Vegetal y Uso de Suelo de la Cuenca Hidrográfica del Canal de Panamá", Panamá, 2013. Disponible en línea: http://www.cich.org/publicaciones/InformeCoberturaVegetal201 3.pdf

[6] David J. Gibson, "Grasses and Grassland Ecology", Oxford University Press, 2009.

[7] Standard Practice for Preparation of Biomass for Compositional Analysis, ASTM E1757-01, 2007.

[8] Standard Test Method for Determination of Total solids in Biomass, ASTM E1756-01, 2001.

[9] Standard Test Method for Ash in Biomass, ASTM E1755-01, 2007.

[10] Junmeng Cai, Yfeng He, Xi Yu, Scott W. Banks, Yang Yang, Xingguang Zhang, Yang Yu, Ronghou Liu, Anthony V. Bridgwater, "Review of physicochemical properties and analytical characterization of lignocellulosic biomass", Elsevier, Renewable and Sustainable Energy Review, vol. 76, pp 309-322, 2017.

[11] Thomas Brackett Reed, Agua Das, Handbook of Biomass Downdraft Gasifier Engine Systems, Solar Energy Research Institute, USA, 1988.

[12] Prabir Basu, Biomass Gasification, Pyrolysis and Torrefaction, Practical Design and Theory, 2nd ed., Academic Press, USA, 2013.
[13] Standard Standard Test Method for Bulk Density of Densified Particulate Biomass Fuels, ASTM E873-82, 1998.

[14] Peter McKendry, "Energy producttion from biomass (part 1): overview of biomass", Elsevier, Bioresource Technology, Vol. 83, Num. 1, pp 37-46, 2001.

[15] Peter McKendry, "Energy production from biomass (part 3): gasification technologies", Elsevier, Bioresource Technology, Vol. 83, Num. 1, pp 55-63, 2002.

[16] Ruprekha Saikia, Rahul Singh Chutia, Rupam Kataki, Kamal K. Pant, "Perennial grass (Arundo donax L.) as a feedstock for thermo-chemical conversion to energy and materials", Elsevier, Bioresource Technology, Vol. 188, pp 265-272, 2015.

[17] Salvatore Luciano Cosentino, Venera Copani, Giorgio Testa, Danilo Scordia, "Saccharum spontaneum L. ssp. (Willd.) Hack. a potencial perennial grass for biomass production in marginal land in semi-arid Mediterranean enviroment", Elsevier, Journal Industrial Crops and Products, Vol. 75, Parte B, pp 93-102, 2015.

[18] Disponible en línea: http://www.hidromet.com.pa/clima_historicos.php?se

\section{I8 1 RIC}

